\title{
Contribution à l'étude des binaires des types F, G, K, M
}

\author{
VIII. HD 195850 et HD 201193, binaires spectroscopiques à raies doubles
}

\section{Contribution to the study of F, G, K, M binaries. VIII. HD 195850 and HD 201193, double-lined spectroscopic binaries}

\author{
J.M. Carquillat et N. Ginestet \\ Observatoire Midi-Pyrénées, Laboratoire d'astrophysique (UMR 5572), 14 avenue Édouard Belin, F-31400 Toulouse, France*
}

Reçu le 21 février; accepté le 23 mars 2000

\begin{abstract}
Radial-velocity observations of the double-lined spectroscopic binaries HD 195850 and HD 201193 have been carried out at the Observatoire de Haute-Provence with the CORAVEL instrument in order to determine their orbital elements. They are as follows • HD 195850: $P=37.94106$ days; $T=2444872.24$ HJD; $\omega=111^{\circ} 5$; $e=0.185 ; K_{1}=34.15 \mathrm{~km} \mathrm{~s}^{-1} ; K_{2}=44.72 \mathrm{~km} \mathrm{~s}^{-1} ; V_{0}=$ $0.75 \mathrm{Km} \mathrm{s}^{-1} ; a_{1} \sin i=17.51 \mathrm{Gm} ; a_{2} \sin i=22.93 \mathrm{Gm}$; $M_{1} \sin ^{3} i=1.04 M_{\odot} ; M_{2} \sin ^{3} i=0.79 M_{\odot}$. • HD 201193: $P=22.299540$ days; $T=2448137.004$ HJD; $\omega=144^{\circ} 7$; $e=0.388 ; K_{1}=54.15 \mathrm{~km} \mathrm{~s}^{-1} ; K_{2}=54.77 \mathrm{~km} \mathrm{~s}^{-1}$; $V_{0}=-13.01 \mathrm{~km} \mathrm{~s}^{-1} ; a_{1} \sin i=15.31 \mathrm{Gm} ; a_{2} \sin i=$ $15.48 \mathrm{Gm} ; M_{1} \sin ^{3} i=1.179 M_{\odot} ; M_{2} \sin ^{3} i=1.165 M_{\odot}$. For HD 195850, we propose a F5V + G2V model on the basis of both the Hipparcos-measured parallax and the $\Delta m_{v}$ value, $1.1 \mathrm{mag}$, deduced from the intensities of the correlation dips. HD 201193, for its part, is formed by two quasi-identical F6V stars. Strömgren photometric indices suggest the following physical parameters: $M_{v}=3.6$; $T_{\text {eff }}=6200 \mathrm{~K} ; \log g=4.2$. Using Schaller et al. (1992) evolutionary tracks for stars of solar metallicity, we estimate $M=1.25 M_{\odot}$ for the mass of each component. This system should be somewhat evolved above the ZAMS, and the possibility of eclipses is, a priori, not excluded; it is likely that the components rotate in synchronism with the orbital motion.
\end{abstract}

Key words: stars: individual: HD 195850; HD 201193 binaries: spectroscopic — stars: fundamental parameters

\footnotetext{
* Étude basée sur des observations effectuées à l'Observatoire de Haute-Provence, France.
}

\section{Introduction}

Les deux étoiles HD 195850 (HIP 100321) et HD 201193 (BD $\left.+35^{\circ} 4402\right)$, suspectées être des binaires spectroscopiques, figuraient à notre programme d'observations systématiques en vitesse radiale (VR) d'étoiles des derniers types spectraux signalées binaires mais sans éléments orbitaux connus.

HD 195850 , étoile de $7^{\mathrm{e}}$ magnitude $(V=7,03)$ dans la constellation du Dragon, était reportée comme binaire spectroscopique dans le General Catalogue of Stellar Radial Velocities (Wilson 1953) : deux clichés spectrographiques, pris à l'observatoire de Lick, avaient mis en évidence une variation de $63 \mathrm{~km} \mathrm{~s}^{-1}$ de sa VR (Moore $\&$ Paddock 1950). Quant à HD 201193 ( $V=7,89)$, de la constellation du Cygne, sa nature binaire spectroscopique nous avait été signalé par J. Figuière (1990) à partir d'observations au prisme objectif faites à l'Observatoire de Haute-Provence (OHP). Cet objet est, de plus, connu comme étoile à fort mouvement propre (Ali 1952).

Nos observations, effectuées au spectrovélocimètre CORAVEL (Baranne et al. 1979) du télescope suisse de 1 mètre de l'OHP, nous ont permis de mettre en évidence la nature binaire spectroscopique à raies doubles (BS2) de ces deux étoiles et d'en déterminer les paramètres orbitaux.

\section{Vitesses radiales et éléments orbitaux}

Pour HD 195850, 52 observations effectuées entre les années 1981 et 1998, s'étendant sur presque 165 révolutions, ont permis le calcul des éléments orbitaux. L'erreur standard interne moyenne sur les VR est de $0,65 \mathrm{~km} \mathrm{~s}^{-1}$ pour la composante primaire et $1,1 \mathrm{~km} \mathrm{~s}^{-1}$ pour la secondaire. 
Pour HD 201193, 47 observations ont été effectuées entre 1990 et 1998, soit une couverture de 133,5 cycles orbitaux. Contrairement au cas précédent, les traces de corrélation sont ici très similaires pour les deux composantes. L'erreur standard interne moyenne est voisine de $0,6 \mathrm{~km} \mathrm{~s}^{-1}$, tant pour les VR de la primaire que pour celles de la secondaire.

Toutes ces VR, données en Tableaux 1 et 2 , sont ramenées au système des standards de l'UAI ; la réduction des observations a été effectuée à l'observatoire de Genève par S. Udry.

Les éléments définitifs de ces deux systèmes ont été calculés avec notre programme Fortran BS2 (Nadal et al. 1979) qui traite globalement les composantes primaire et secondaire des binaires à deux spectres. Les pondérations suivantes ont été appliquées : pour HD 195850, 1 pour les VR de la primaire et $1 / 4$ pour celles de la secondaire ; pour HD 201193, 1 pour toutes les VR.

Les éléments orbitaux obtenus sont donnés ci-dessous, les résidus $\mathrm{O}-\mathrm{C}$ en Tableaux 1 et 2 et les courbes de VR calculées aux Figs. 1 et 2.

\begin{tabular}{lcc}
\hline Eléments & HD 195850 & HD 201 193 \\
\hline$P$ (jours) & $37,94106 \pm 0,00055$ & $22,299540 \pm 0,000085$ \\
$T$ JJ $2400000+$ & $44872,24 \pm 0,10$ & $48137,004 \pm 0,011$ \\
$\omega$ (degrés) & $111,5 \pm 0,9$ & $144,7 \pm 0,2$ \\
$e$ & $0,185 \pm 0,003$ & $0,388 \pm 0,001$ \\
$K_{1}\left(\mathrm{~km} \mathrm{~s}^{-1}\right)$ & $34,15 \pm 0,10$ & $54,15 \pm 0,10$ \\
$K_{2}\left(\mathrm{~km} \mathrm{~s}^{-1}\right)$ & $44,72 \pm 0,20$ & $54,77 \pm 0,10$ \\
$V_{0}\left(\mathrm{~km} \mathrm{~s}^{-1}\right)$ & $0,75 \pm 0,07$ & $-13,01 \pm 0,05$ \\
$a_{1} \sin i(\mathrm{Gm})$ & $17,51 \pm 0,05$ & $15,31 \pm 0,03$ \\
$a_{2} \sin i(\mathrm{Gm})$ & $22,93 \pm 0,10$ & $15,48 \pm 0,03$ \\
$M_{1} \sin ^{3} i\left(M_{\odot}\right)$ & $1,04 \pm 0,01$ & $1,179 \pm 0,005$ \\
$M_{2} \sin ^{3} i\left(M_{\odot}\right)$ & $0,79 \pm 0,01$ & $1,165 \pm 0,005$ \\
$\sigma(\mathrm{O}-\mathrm{C})\left(\mathrm{km} \mathrm{s}^{-1}\right)$ & 0,53 & 0,47 \\
$\cdots$ & &
\end{tabular}

\section{Discussion}

\section{1. $H D 195850$}

Afin de proposer un modèle pour le système nous sommes partis des données suivantes :

- classification spectrale F5IV de Moore \& Paddock (1950), effectuée sur des spectres photographiques à $75 \AA / \mathrm{mm}$ de dispersion ;

- parallaxe $\pi=0^{\prime \prime}, 184$ (soit $d=54 \mathrm{pc}$ ) mesurée par Hipparcos (avec une très bonne précision : $\sigma_{\pi} / \pi=0,03$ ) ;

- magnitude $V=7,03$ et indice $B-V=0,46$ de Tycho ramenés dans le système de Johnson (ESA 1997). À noter que ces valeurs sont très proches de celles $(V=7,05 ; B-V=0,45)$ obtenues par Wesselink (1989) en réduisant dans le système $U, B, V$ des magnitudes photographiques. Par contre, la magnitude $V$ apparaît

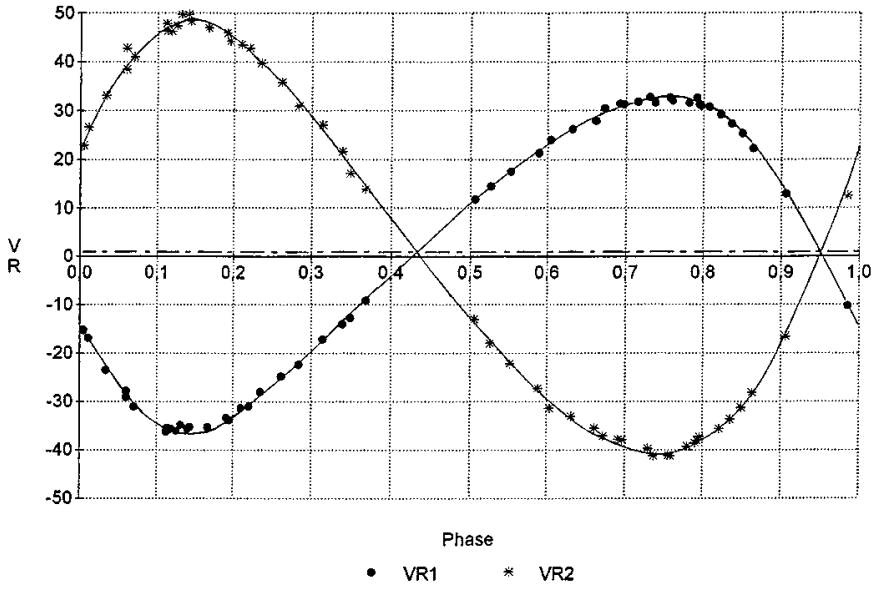

Fig. 1. Courbes de vitesse radiale de HD 195850. Cercles pleins : composante primaire ; astérisques : composante secondaire. L'origine des phases est le passage au périastre

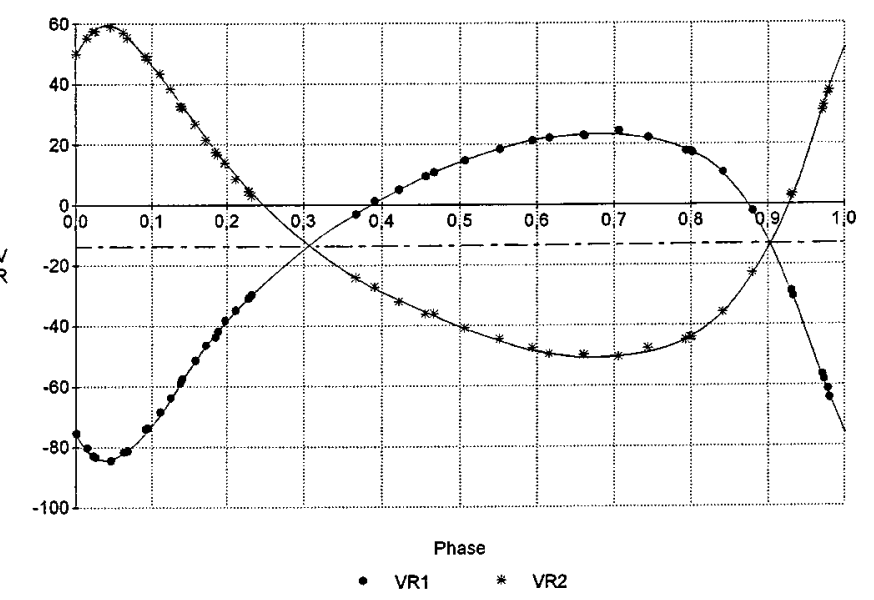

Fig. 2. Courbes de vitesse radiale de HD 201193. Cercles pleins : composante primaire ; astérisques : composante secondaire. L'origine des phases est le passage au périastre

notablement différente de la magnitude visuelle 8,1 donnée pour HD 195850 dans le Henry Draper Catalogue. Sans cette discordance, la nature binaire de cette étoile serait peut-être encore méconnue, car l'échantillon étudié par Moore \& Paddock, dont les observations ont permis de suspecter cette binarité, ne comportait que des étoiles voisines de la $8^{\mathrm{e}}$ magnitude !

- différence de magnitude entre les composantes du couple que l'on peut déduire du rapport des largeurs équivalentes des traces de corrélation (Lucke \& Mayor 1980). Si, dans un premier temps, on prend $T_{1} / T_{2} \sim 1$, $T_{1}$ et $T_{2}$ étant les températures effectives de la primaire et de la secondaire, on obtient $\Delta m_{b}=1,4$;

- rapport de masses $\rho=0,76$ déduit des paramètres orbitaux.

Étant donné les valeurs de $\pi$ et de $V$ pour le système, on doit avoir $M v_{1}>3,35$ pour la primaire, ce qui indiquerait que sa classe de luminosité n'est pas IV mais V (Schmidt-Kaler 1982). Dans la suite de cette 
Tableau 1. Vitesses radiales de HD $195850: \mathrm{VR}_{1},(\mathrm{O}-\mathrm{C})_{1}$, vitesses et résidus pour la primaire ; $\mathrm{VR}_{2},(\mathrm{O}-\mathrm{C})_{2}$, mêmes quantités pour la secondaire. Les phases sont données par rapport au passage au périastre

\begin{tabular}{|c|c|c|c|c|c|c|c|}
\hline $\mathrm{N}^{\circ}$ & Date & $\begin{array}{c}\text { Date JJH } \\
2400000+ \\
\end{array}$ & Phase & $\begin{array}{l}\mathrm{VR}_{1} \\
\mathrm{~km} \mathrm{~s}^{-1} \\
\end{array}$ & $\begin{array}{c}(\mathrm{O}-\mathrm{C})_{1} \\
\mathrm{~km} \mathrm{~s}^{-1}\end{array}$ & $\begin{array}{l}\mathrm{VR}_{2} \\
\mathrm{~km} \mathrm{~s}^{-1} \\
\end{array}$ & $\begin{array}{c}(\mathrm{O}-\mathrm{C})_{2} \\
\mathrm{~km} \mathrm{~s}^{-1}\end{array}$ \\
\hline 1 & 11.09 .1981 & 44859,426 & 0,662 & $+27,9$ & $-1,2$ & $-35,6$ & $+0,8$ \\
\hline 2 & 13. & 44861,410 & 0,715 & $+31,8$ & $-0,2$ & & \\
\hline 3 & 16. & 44864,438 & 0,794 & $+31,3$ & $+0,1$ & $-38,1$ & $+1,1$ \\
\hline 4 & 17. & 44865,469 & 0,822 & $+29,2$ & $+0,2$ & $-35,7$ & $+0,5$ \\
\hline 5 & 23.08 .1982 & 45204,574 & 0,759 & $+32,0$ & $-0,5$ & $-41,3$ & $-0,4$ \\
\hline 6 & 17.09 .1983 & 45595,402 & 0,060 & $-27,8$ & $+1,0$ & $+42,7$ & $+3,2$ \\
\hline 7 & 19. & 45597,387 & 0,112 & $-36,2$ & $-1,2$ & $+47,8$ & $+0,2$ \\
\hline 8 & 19. & 45597,406 & 0,113 & $-35,4$ & $-0,3$ & $+46,3$ & $-1,3$ \\
\hline 9 & 20. & 45598,422 & 0,140 & $-35,6$ & $+0,1$ & $+49,7$ & $+1,2$ \\
\hline 10 & 22. & 45600,348 & 0,191 & $-33,3$ & $+0,1$ & $+45,9$ & $+0,4$ \\
\hline 11 & 10.11 .1984 & 46015,430 & 0,131 & $-34,8$ & $+0,9$ & $+49,7$ & $+1,3$ \\
\hline 12 & 27.09 .1985 & 46336,340 & 0,589 & $+21,4$ & $-0,8$ & $-27,3$ & $+0,1$ \\
\hline 13 & 17.10 .1986 & 46721,359 & 0,737 & $+31,6$ & $-0,9$ & $-41,3$ & $-0,4$ \\
\hline 14 & 19. & 46723,438 & 0,791 & $+32,7$ & $+1,3$ & $-38,6$ & $+0,8$ \\
\hline 15 & 29.10 .1987 & 47098,352 & 0,673 & $+30,5$ & $+0,6$ & $-37,2$ & $+0,2$ \\
\hline 16 & 11.12 .1989 & 47872,250 & 0,070 & $-31,0$ & $-0,4$ & $+41,0$ & $-0,7$ \\
\hline 17 & 13. & 47874,313 & 0,125 & $-36,0$ & $-0,5$ & $+47,4$ & $-0,8$ \\
\hline 18 & 25.08 .1990 & 48129,473 & 0,850 & $+25,3$ & $-0,0$ & $-31,3$ & $+0,2$ \\
\hline 19 & 31. & 48135,344 & 0,005 & $-15,2$ & $+0,2$ & $+22,9$ & $+1,0$ \\
\hline 20 & 01.09 .1990 & 48136,461 & 0,034 & $-23,5$ & $-0,2$ & $+33,1$ & $+0,9$ \\
\hline 21 & 02 & 48137,453 & 0,060 & $-29,1$ & $-0,2$ & $+38,4$ & $-1,1$ \\
\hline 22 & 09.11 .1992 & 48936,391 & 0,118 & $-35,6$ & $-0,3$ & $+46,2$ & $-1,7$ \\
\hline 23 & 10 & 48937,359 & 0,143 & $-35,2$ & $+0,5$ & $+48,3$ & $-0,2$ \\
\hline 24 & 12. & 48939,313 & 0,195 & $-33,8$ & $-0,7$ & $+44,2$ & $-0,8$ \\
\hline 25 & 13. & 48940,336 & 0,221 & $-30,9$ & $-0,5$ & $+42,8$ & $+1,3$ \\
\hline 26 & 09.12 .1992 & 48966,316 & 0,906 & $+12,9$ & $-0,8$ & $-16,6$ & $-0,3$ \\
\hline 27 & 12. & 48969,297 & 0,985 & $-10,2$ & $-0,7$ & $+12,4$ & $-1,7$ \\
\hline 28 & 13. & 48970,281 & 0,011 & $-16,8$ & $+0,4$ & $+26,6$ & $+2,4$ \\
\hline 29 & 03.06 .1993 & 49141,586 & 0,526 & $+14,5$ & $+0,0$ & $-18,0$ & $-0,8$ \\
\hline 30 & 04 & 49142,578 & 0,552 & $+17,5$ & $-0,4$ & $-22,2$ & $-0,5$ \\
\hline 31 & 06. & 49144,559 & 0,604 & $+24,1$ & $+0,2$ & $-31,4$ & $-1,8$ \\
\hline 32 & 07. & 49145,613 & 0,632 & $+26,2$ & $-0,4$ & $-33,1$ & $+0,0$ \\
\hline 33 & 27.11 .1993 & 49319,309 & 0,210 & $-31,3$ & $+0,3$ & $+43,5$ & $+0,4$ \\
\hline 34 & 28. & 49320,309 & 0,236 & $-28,0$ & $+0,6$ & $+39,7$ & $+0,5$ \\
\hline 35 & 29. & 49321,289 & 0,262 & $-24,7$ & $+0,5$ & $+35,8$ & $+1,1$ \\
\hline 36 & 01.12 .1993 & 49323,273 & 0,314 & $-17,1$ & $+0,4$ & $+27,0$ & $+2,4$ \\
\hline 37 & 03. & 49325,301 & 0,368 & $-9,1$ & $-0,0$ & $+13,8$ & $+0,2$ \\
\hline 38 & 15.10 .1994 & 49641,320 & 0,697 & $+31,3$ & $+0,0$ & $-38,0$ & $+1,2$ \\
\hline 39 & 30.08 .1996 & 50325,512 & 0,730 & $+32,8$ & $+0,4$ & $-39,8$ & $+0,9$ \\
\hline 40 & 31. & 50326,500 & 0,756 & $+32,7$ & $+0,1$ & $-41,2$ & $-0,3$ \\
\hline 41 & 31. & 50327,453 & 0,781 & $+31,6$ & $-0,3$ & $-39,3$ & $+0,7$ \\
\hline 42 & 01.09 .1996 & 50328,426 & 0,807 & $+30,8$ & $+0,5$ & & \\
\hline 43 & 03. & 50329,520 & 0,836 & $+27,3$ & $-0,0$ & $-33,7$ & $+0,4$ \\
\hline 44 & 15.06 .1997 & 50614,594 & 0,349 & $-12,7$ & $-0,7$ & $+17,1$ & $-0,3$ \\
\hline 45 & 19.10 .1997 & 50741,414 & 0,692 & $+31,4$ & $+0,4$ & $-37,9$ & $+1,0$ \\
\hline 46 & 23 & 50745,414 & 0,797 & $+31,0$ & $-0,0$ & $-37,4$ & $+1,5$ \\
\hline 47 & 24. & 50746,352 & 0,822 & $+29,3$ & $+0,4$ & & \\
\hline 48 & 27.12 .1997 & 50810,246 & 0,506 & $+11,8$ & $+0,0$ & $-13,1$ & $+0,6$ \\
\hline 49 & 21.01 .1998 & 50835,297 & 0,166 & $-35,2$ & $-0,2$ & $+46,9$ & $-0,7$ \\
\hline 50 & 11.06 .1998 & 50975,605 & 0,864 & $+22,3$ & $-0,6$ & $-28,2$ & $+0,1$ \\
\hline 51 & 18.10 .1998 & 51105,363 & 0,284 & $-22,3$ & $-0,3$ & $+30,9$ & $+0,3$ \\
\hline 52 & 20. & 51107,426 & 0,339 & $-14,0$ & $-0,3$ & $\begin{array}{r}+21,6 \\
\end{array}$ & $+2,0$ \\
\hline
\end{tabular}

investigation, nous retiendrons donc le type spectral F5V pour la composante principale. Compte tenu de la valeur initiale de $\Delta m_{b}$ nous avons d'abord attribué le type G3V à la secondaire. À l'issue d'une "itération", en faisant intervenir cette fois le rapport $T_{1} / T_{2}$ pour l'estimation de $\Delta m_{b}$, ce type spectral était ramené à $\mathrm{G} 2 \mathrm{~V}$ avec $\Delta m_{b}=1,3$, soit $\Delta m_{v}=1,1$.

Dans le tableau ci-dessous, nous confrontons les paramètres observés à ceux déduits des calibrations de
Schmidt-Kaler (1982) et des données d'Andersen (1990) pour le modèle $\mathrm{F} 5 \mathrm{~V}+\mathrm{G} 2 \mathrm{~V}$ proposé :

\begin{tabular}{|c|c|c|c|c|c|c|}
\hline Paramètres & $M_{l} / M_{\odot}$ & $M_{2} / M_{\odot}$ & $\rho$ & $M_{v}$ & $\Delta m_{v}$ & $B-V$ \\
\hline Modèle & 1,3 & 1,0 & 0,75 & 3,2 & 1,2 & 0,48 \\
\hline Observé & & & 0,76 & 3,35 & 1,1 & 0,46 \\
\hline
\end{tabular}


Tableau 2. Vitesses radiales de HD $201193: \mathrm{VR}_{1},(\mathrm{O}-\mathrm{C})_{1}$, vitesses et résidus pour la primaire ; $\mathrm{VR}_{2},(\mathrm{O}-\mathrm{C})_{2}$, mêmes quantités pour la secondaire. Les phases sont données par rapport au passage au périastre

\begin{tabular}{|c|c|c|c|c|c|c|c|}
\hline $\mathrm{N}^{\circ}$ & Date & $\begin{array}{c}\text { Date JJH } \\
24000000+\end{array}$ & Phase & $\begin{array}{l}\mathrm{VR}_{1} \\
\mathrm{~km} \mathrm{~s}^{-1}\end{array}$ & $\begin{array}{l}(\mathrm{O}-\mathrm{C})_{1} \\
\mathrm{~km} \mathrm{~s}^{-1}\end{array}$ & $\begin{array}{l}\mathrm{VR}_{2} \\
\mathrm{~km} \mathrm{~s}^{-1}\end{array}$ & $\begin{array}{c}(\mathrm{O}-\mathrm{C})_{2} \\
\mathrm{~km} \mathrm{~s}^{-1}\end{array}$ \\
\hline 1 & 24.08 .1990 & 48128,449 & 0,616 & $+22,1$ & $-0,1$ & $-49,4$ & $-0,7$ \\
\hline 2 & 25. & 48129,461 & 0,662 & $+22,7$ & $-1,0$ & $-49,8$ & $+0,3$ \\
\hline 3 & 28. & 48132,523 & 0,799 & $+17,7$ & $-0,0$ & $-44,1$ & $+0,0$ \\
\hline 4 & 29. & 48133,465 & 0,841 & $+10,7$ & $+0,9$ & $-35,9$ & $+0,2$ \\
\hline 5 & 31. & 48135,496 & 0,932 & $-30,4$ & $-0,4$ & $+3,3$ & $-0,9$ \\
\hline 6 & 02.09 .1990 & 48136,504 & 0,978 & $-61,1$ & $+0,2$ & $+36,4$ & $+0,6$ \\
\hline 7 & 02. & 48137,348 & 0,015 & $-80,2$ & $+0,3$ & $+55,2$ & $-0,0$ \\
\hline 8 & 03. & 48137,594 & 0,026 & $-83,2$ & $-0,1$ & $+57,4$ & $-0,5$ \\
\hline 9 & 12.11 .1992 & 48939,340 & 0,980 & $-64,2$ & $-1,4$ & $+37,5$ & $+0,1$ \\
\hline 10 & 13. & 48940,293 & 0,023 & $-82,8$ & $-0,5$ & $+57,5$ & $+0,4$ \\
\hline 11 & 14. & 48941,293 & 0,068 & $-81,2$ & $+0,0$ & $+55,3$ & $-0,7$ \\
\hline 12 & 09.12 .1992 & 48966,289 & 0,188 & $-41,8$ & $-0,0$ & $+16,6$ & $+0,5$ \\
\hline 13 & 10. & 48967,279 & 0,233 & $-29,6$ & $-0,4$ & $+3,0$ & $-0,5$ \\
\hline 14 & 13. & 48970,266 & 0,367 & $-3,0$ & $-0,7$ & $-24,1$ & $-0,3$ \\
\hline 15 & 15 & 48972,254 & 0,456 & $+9,6$ & $+0,1$ & $-36,2$ & $-0,4$ \\
\hline 16 & 14.01 .1993 & 49002,246 & 0,801 & $+17,2$ & $-0,3$ & $-44,0$ & $-0,1$ \\
\hline 17 & 04.06 .1993 & 49142,605 & 0,095 & $-73,6$ & $-0,2$ & $+48,0$ & $-0,1$ \\
\hline 18 & 05 & 49143,617 & 0,141 & $-57,2$ & $+0,4$ & $+32,0$ & $-0,1$ \\
\hline 19 & 06. & 49144,602 & 0,185 & $-43,6$ & $-0,6$ & $+17,4$ & $+0,1$ \\
\hline 20 & 07. & 49145,598 & 0,229 & $-30,7$ & $-0,5$ & $+4,5$ & $+0,1$ \\
\hline 21 & 25.11 .1993 & 49317,309 & 0,930 & $-28,7$ & $-0,5$ & $+2,7$ & $+0,3$ \\
\hline 22 & 26. & 49318,281 & 0,973 & $-58,0$ & $+0,3$ & $+32,6$ & $-0,2$ \\
\hline 23 & 28. & 49320,281 & 0,063 & $-81,6$ & $+0,5$ & $+57,0$ & $+0,1$ \\
\hline 24 & 29. & 49321,344 & 0,111 & $-68,3$ & $-0,1$ & $+43,3$ & $+0,4$ \\
\hline 25 & 01.12 .1993 & 49323,305 & 0,198 & $-38,2$ & $+0,6$ & $+13,9$ & $+0,8$ \\
\hline 26 & 14.10 .1994 & 49640,484 & 0,422 & $+5,2$ & $-0,3$ & $-32,1$ & $-0,4$ \\
\hline 27 & 15 & 49641,484 & 0,467 & $+10,9$ & $+0,2$ & $\begin{array}{l}-36,2 \\
\end{array}$ & $+0,8$ \\
\hline 28 & 16. & 49642,352 & 0,506 & $+14,8$ & $+0,2$ & $-40,9$ & $+0,0$ \\
\hline 29 & 17. & 49643,352 & 0,551 & $+18,5$ & $+0,2$ & $-44,5$ & $+0,2$ \\
\hline 30 & 18. & 49644,309 & 0,594 & $+21,3$ & $+0,2$ & $-47,4$ & $+0,1$ \\
\hline 31 & 18.04 .1996 & 50191,641 & 0,138 & $-58,7$ & $-0,2$ & $+32,6$ & $-0,4$ \\
\hline 32 & 28.08 .1996 & 50324,410 & 0,092 & $-74,0$ & $+0,5$ & $+49,1$ & $-0,1$ \\
\hline 33 & 29. & 50325,488 & 0,140 & $-57,7$ & $-0,0$ & $+32,6$ & $+0,4$ \\
\hline 34 & 31. & 50327,480 & 0,230 & $-30,5$ & $-0,4$ & $+4,6$ & $+0,3$ \\
\hline 35 & 26.11 .1996 & 50414,336 & 0,125 & $-63,7$ & $-0,5$ & $+38,4$ & $+0,6$ \\
\hline 36 & 27. & 50415,402 & 0,172 & $-46,3$ & $+0,5$ & $+21,4$ & $+0,2$ \\
\hline 37 & 28 & 50416,281 & 0,212 & $\begin{array}{l}-34,7 \\
\end{array}$ & $+0,2$ & $+8,5$ & $-0,7$ \\
\hline 38 & 02.12 .1996 & 50420,277 & 0,391 & $+1,5$ & $+0,2$ & $-27,2$ & $+0,3$ \\
\hline 39 & 10.06.1997 & 50609,586 & 0,880 & $-2,1$ & $+0,6$ & $-22,7$ & $+0,7$ \\
\hline 40 & 12. & 50611,605 & 0,971 & $-56,5$ & $+0,3$ & $+30,9$ & $-0,4$ \\
\hline 41 & 16.10 .1997 & 50738,480 & 0,661 & $+23,0$ & $-0,7$ & $-49,9$ & $+0,2$ \\
\hline 42 & 18 . & 50739,500 & 0,706 & $+24,4$ & $+0,5$ & $-50,5$ & $-0,2$ \\
\hline 43 & 18. & 50740,352 & 0,744 & $+22,2$ & $-0,5$ & $-47,7$ & $+1,4$ \\
\hline 44 & 19. & 50741,434 & 0,793 & $+17,8$ & $-0,7$ & $-45,0$ & $-0,1$ \\
\hline 45 & 21.01 .1998 & 50835,273 & 0,001 & $-75,3$ & $-0,4$ & $+49,9$ & $+0,3$ \\
\hline 46 & 22 & 50836,270 & 0,046 & $-84,4$ & $-0,2$ & $+58,9$ & $-0,1$ \\
\hline 47 & 19.10 .1998 & 51106,359 & 0,158 & $-51,2$ & $+0,5$ & $+26,7$ & $+0,6$ \\
\hline
\end{tabular}

Inclinaison orbitale, séparation des composantes

À partir de la masse attribuée à la primaire, 1,3 $M_{\odot}$, la valeur de $M_{1} \sin ^{3} i$ donne $i=68^{\circ}$, et la séparation des composantes peut être estimée à : $a=a_{1}+a_{2}=$ 43,56 $\mathrm{Gm}=0,3 \mathrm{UA}=63 R_{\odot}$. La possibilité d'éclipses semble donc exclue pour ce système.

\section{Rotation, synchronisme}

L'analyse du profil des traces de corrélation obtenues à CORAVEL permet d'estimer les vitesses de rotation $v \cdot \sin i$ des composantes du système (méthode décrite par Benz \& Mayor 1981), ce qui donne ici : 13,3 $\pm 1,0 \mathrm{~km} \mathrm{~s}^{-1}$ pour la primaire et $3,5 \pm 1,0 \mathrm{~km} \mathrm{~s}^{-1}$ pour la secondaire. À titre de comparaison, les valeurs des vitesses de rotation équatoriale dans le cas de synchronisme rotationrévolution, en attribuant aux composantes les rayons correspondant aux types spectraux envisagés (Schmidt-Kaler 1982), seraient de $1,7 \mathrm{~km} \mathrm{~s}^{-1}$ et $1,3 \mathrm{~km} \mathrm{~s}^{-1}$ pour la primaire et la secondaire respectivement. Le stade du "synchronisme" n'est donc vraisemblablement pas atteint pour HD 195850.

\section{2. $H D 201193$}

Comme nous l'avons déjà signalé, les composantes de cette BS2 présentent des traces de corrélation similaires : 
HD 201193 est composée de deux étoiles quasi identiques, ainsi que le confirme le rapport de masses pratiquement égal à l'unité $(\rho=0,99) ; \Delta m=0$.

Pour la classification de cette étoile, nous ne disposions que de celle, F8, donnée dans le Henry Draper Catalogue et retenue dans la base SIMBAD du Centre de Données Astronomiques de Strasbourg. Afin d'obtenir une classification dans le système MK, nous avons observé HD 201193 au spectrographe AURELIE de l'OHP, au cours de notre programme de classification des composantes chaudes des binaires à spectre composite ; nous avons eu l'opportunité d'observer le système à une phase où les composantes avaient une VR proche de $V_{0}$ et où par conséquent les raies apparaissent simples. La comparaison du spectre obtenu à celui des étoiles standards MK prises avec le même instrument indique que les composantes de HD 201193 sont d'un type voisin de F6 V.

\section{Paramètres physiques}

HD 201193 n'a pas été observée par le satellite Hipparcos, et nous ne pouvons donc pas estimer directement sa magnitude absolue. Néanmoins, nous pouvons utiliser les indices de la photométrie de Strömgren pour cet objet (Olsen 1983) comme ceux d'une étoile simple étant donné la nature similaire des deux composantes : $b-y=0,342$; $m_{1}=0,150 ; c_{1}=0,409 ; \beta=2,626 ; V=7,89$.

Ces quantités nous permettent l'estimation de certains paramètres physiques fondamentaux des deux étoiles constituant le système : à partir des calibrations de Crawford (1975), on obtient en effet $\delta m_{1}=0,039$ et $\delta c_{1}=0,059$, ce qui conduit à $M_{v}=3,6$ et $[\mathrm{Fe} / \mathrm{H}]$ $=-0,2$ dex, et, en utilisant la grille de Moon \& Dworetsky (1985) pour les étoiles plus froides que $8500 \mathrm{~K}$, $T_{\text {eff }} \cong 6200 \mathrm{~K}$ et $\log g \cong 4,2$. Nous pouvons en déduire $M_{\mathrm{bol}}=3,5 ; \log \left(L / L_{\odot}\right)=0,5$ et $R=1,6 R_{\odot}$.

\section{Masses, état d'évolution}

En utilisant le diagramme HR théorique de Schaller et al. (1992), $M_{\text {bol }}$ vs. $\log T_{\text {eff }}$, pour les étoiles de métallicité solaire, nous pouvons estimer la masse des composantes de HD 201193 à 1,25 $M_{\odot}$ ainsi que leur état d'évolution : ces étoiles apparaissent être des naines déjà évoluées sur la séquence principale, de quelque 0,7 mag au-dessus de la ZAMS, ce que laissait prévoir la valeur relativement élevée du rayon trouvé ci-dessus.

La formule : $\log g=\log \left(M / M_{\odot}\right)+4 \log \left(T / T_{\odot}\right)-$ $\log \left(L / L_{\odot}\right)+4,44$ donne ici $\log g=4,14$, valeur très proche de celle déduite, plus haut, de la photométrie de Strömgren.

La valeur présumée de la masse implique $i=79^{\circ}$ et une séparation moyenne des composantes $a=0,2$
$\mathrm{UA}=45 R_{\odot}$. La possibilité d'éclipses n'apparaît pas exclue a priori si l'on considère que l'écart entre la masse "théorique" et $M_{1} \sin ^{3} i$ n'est que de $0,07 M_{\odot}$ !

\section{Rotation, synchronisme}

La vitesse de rotation $v \cdot \sin i$, obtenue par l'analyse des traces de corrélation, apparaît faible pour les composantes du système, étant donné leur type spectral : 3,0 \pm $1,0 \mathrm{~km} \mathrm{~s}^{-1}$ pour la primaire et $2,5 \pm 1,0 \mathrm{~km} \mathrm{~s}^{-1}$ pour la secondaire. $\mathrm{Vu}$ la relativement courte période orbitale de 22 jours, on peut penser que le phénomène de synchronisme rotation-révolution a pu contribuer a freiner la rotation axiale des composantes. En effet, si on prend $R=1,6 R_{\odot}$ (voir plus haut), la vitesse équatoriale des composantes serait de $3,6 \mathrm{~km} \mathrm{~s}^{-1}$. Inversement, compte tenu de la valeur présuméé de $i$ et en supposant la coplanarité, les valeurs de $v \cdot \sin i$ conduiraient a des rayons de $1,36 R_{\odot}$ pour la primaire et de $1,14 R_{\odot}$ pour la secondaire, quantités compatibles avec le type spectral retenu. L'hypothèse du synchronisme apparaît donc plausible.

Remerciements. Nous tenons à exprimer notre gratitude aux Drs. Michel Mayor et Stéphane Udry pour l'utilisation de l'instrument Coravel et la réduction des observations ; merci également au Dr. Roger Griffin pour une observation de HD 195850 effectuée au cours de la nuit du 27.12.1997.

\section{Bibliographie}

Ali A., 1952, J. Obs. 35, 129

Andersen J., 1991, A\&AR 3, 91

Baranne A., Mayor M., Poncet J.L., 1979, Vistas Astron. 23, 279

Benz W., Mayor M., 1981, A\&A 93, 235

Crawford D.L., 1975, AJ 80, 955 ESA, 1997, The Hipparcos and Tycho Catalogues, ESA SP-1200

Figuière J., 1990 (communication privée)

Lucke P.B., Mayor M., 1980, A\&A 92, 182

Moon T.T., Dworetsky M.M., 1985, MNRAS 217, 305

Moore J.H., Paddock G.F., 1950, ApJ 112, 48

Nadal R., Ginestet N., Carquillat J.M., Pédoussaut A., 1979, A\&AS 35, 203

Olsen E.H., 1983, A\&AS 54, 55

Schaller G., Schaerer D., Meynet G., Maeder A., 1992, A\&AS 96, 269

Schmidt-Kaler Th., 1982, in: Landolt-Börnstein, New Series, Gr. 6, Vol. 2-B. Springer-Verlag, Berlin, Heidelberg, New-York, p. 1

Wesselink, 1989, Observatory 109, 5

Wilson R.E., 1953, General Catalogue of Stellar Radial Velocities, Carnegie Institution Washington Publ. 601 